\title{
Editorial
}

\section{La imperiosa necesidad de reconstruir el sistema valorativo de los aportes científicos}

Desde hace ya varios años, la comunidad científica se desenvuelve en una situación de crisis relacionada con los hallazgos científicos, en particular con aquellos concernientes a la esfera de la salud. Cuatro ejes, mutuamente condicionados, producen un genuino estado de alarma:

en primer lugar, se vive una crisis de credibilidad, que se hizo patente mediante los aportes de John Ioannidis, profesor de la Universidad de Stanford. En 2005, este médico y epistemólogo greconorteamericano sacudió al mundo de la investigación y las publicaciones en salud con un artículo [1] que mantiene plena vigencia y que, desde su propio título, da por sentado que la mayoría de los hallazgos de las publicaciones científicas contemporáneas arriban a conclusiones incorrectas. De hecho, pasó a ser el artículo científico más veces descargado de la Web desde entonces, en tanto que su autor es el médico más citado del mundo.

Complementariamente, nos hallamos en medio de una aguda crisis de reproducibilidad, como se ha advertido desde hace algunos años [2]. La replicación de un resultado se produce cuando este se confirma en estudios subsiguientes realizados en condiciones similares. A pesar de que este aval constituye uno de los pilares más importantes para el progreso científico, una abrumadora mayoría de los investigadores sostiene que la ciencia contemporánea experimenta una insolvencia crítica en esta materia. Según una reciente encuesta aplicada a casi 1600 investigadores por la influyente revista Nature, solo el $3 \%$ consideró que no existía tal crisis [3].

El otro mecanismo llamado a garantizar la calidad de las contribuciones científicas es la llamada "revisión por pares" (peer review, en inglés). Se trata de una práctica implantada desde 1900 en procura de evitar la diseminación de trabajos metodológicamente endebles o carentes de la debida sustentación, que proliferaban hasta entonces. Tal garantía se ha visto crecientemente comprometida [4], en buena medida por la inescrupulosa invasión de conflictos de interés [5] que condicionan al mundo salubrista. A ocho años desde que se lanzara la loable "Iniciativa de Budapest" [6], poco se ha concretado de lo que se ha reclamado [7] para consumar la idea de contar con políticas de acceso a una comunicación científica sostenible, confiable y no condicionada por el mercado.

A lo anterior se agregan, finalmente, los consabidos recelos que despierta la dictadura del "factor de impacto" [8], indicador bibliométrico impuesto por la transnacional Thomson Reuter para medir la calidad de las revistas, que están lejos de disiparse.

En este contexto, aparece la pandemia asociada al CovID-19, que ha venido reconfigurando la realidad en virtualmente todas las esferas de la sociedad. La problemática que nos ocupa no es una excepción.

El apremio por controlar la epidemia y minimizar sus efectos es natural y justificado. Pero el afán de lucro y la lucha por conseguir prominencia mediática han añadido elementos especialmente inquietantes al conflictivo panorama arriba bosquejado. Abundan manifestaciones de ese nefasto efecto.

Bastaría reparar en el rocambolesco laberinto de comunicaciones [9] relacionadas con el remdesivir, el famoso antiviral agresivamente publicitado por la compañía biotecnológica Gilead Sciences y detrás de las cuales danzan decenas de millones de dólares. Pero examinemos, aunque sea someramente, el caso de la hidroxicloroquina, protagonista del mayor escándalo científico de la pandemia. El frenesí publicador desatado en torno a este fármaco antipalúdico, comercializado por Sanofi, pone al desnudo el galopante deterioro del sistema valorativo de los aportes científicos que urge reconstruir.

La catarata de despropósitos editoriales se inicia con la publicación de un "preprint" (manuscrito no revisado por pares), el cual erigía a la hidroxicloroquina poco menos que a la condición de panacea terapéutica [10]. Aprobado en solo 24 horas y con notables fallos de diseño, pronto afloraron más recelos que esperanzas. Sin embargo, la insólita e insistente promoción del fármaco a cargo de dos jefes de Estado (los presidentes Jair Bolsonaro y Donald Trump) avivaron el interés, y el precio de las acciones de Sanofi aumentó en un $16 \%$ [11]. 
Unas semanas después, la encumbrada revista británica The Lancet — la más importante de las 2500 revistas científicas controladas por el grupo oligopólico Elsevier - se precipitó a publicar, sin la debida valoración de su validez, los resultados de un enorme estudio (96000 pacientes ingresados en 671 hospitales de seis continentes). Fue una bomba de relojería que atribuía al fármaco una notable responsabilidad por la muerte prematura de muchos de sus consumidores [12]. De inmediato, la explosión: varios países prohibieron el uso del fármaco y, al igual que la Organización Mundial de la Salud (OMS), detuvieron los ensayos clínicos que intentaban demostrar su eficacia. Otra vez la verdad científica estaba siendo sacrificada en el altar de una conducta editorial irresponsable. No demoró la demostración de que se trataba de un fraude, admitido por la propia revista y por tres de sus cuatro autores. La oms, que originalmente había convalidado su empleo, pidió disculpas por haberse plegado a tal desaguisado y retomó el ensayo clínico que había suspendido pocos días antes. La escalada de marchas y contramarchas editoriales siguió creciendo. El último episodio ha sido el muy reciente informe de la oms, "Repurposed antiviral drugs for COVID-19-interim wHO SOLIDARITY trial results" [13], el cual revela que su voluminoso ensayo clínico — ahora concluido - no convalida la proclamada capacidad del fármaco para reducir el riesgo de muerte de los enfermos, gracias a la cual la farmacéutica Sanofi ha tenido millonarias ganancias.

En cierto sentido, las controversias pueden considerarse normales e incluso entenderse como saludable expresión de la vocación autocrítica de la ciencia. Lo que resulta inadmisible, sin embargo, es que ellas se deriven de un menoscabo flagrante del rigor editorial.

El sistema de publicaciones científicas reclama urgente remodelación, que empiece por incentivar y recuperar, sin ambages, la práctica de la revisión por pares. Pero, a ese empeño, deberían añadirse al menos las siguientes dos acciones: por una parte, establecer "unidades de integridad científica" en las universidades, órganos que velen por que sus académicos cumplan las demandas éticas, metodológicas y de transparencia reclamadas en los ámbitos científicamente reputados; y, por otra, promover espacios que permitan supervisar y valorar el cumplimiento de esos mismos atributos por parte de las contribuciones ya publicadas. La más connotada de estas iniciativas ha sido impulsada por la Fundación PubPeer, que ha creado un exitoso espacio interactivo (http://www.PubPeer.com), donde cualquier investigador puede participar en la revisión por pares después de la publicación.

Dr. Luis Carlos Silva Aycaguer

PhD en Ciencias Matemáticas y en Ciencias de la Salud

Editorialista invitado

\section{Referencias}

1. Ioannidis JP. Why most published research findings are false. PLos Medicine. 2005;2(8):0696-0701. DoI: https://doi.org/10.1371/journal. pmed.0020124

2. Begley CG, Ioannidis JP. Reproducibility in science: Improving the standard for basic and preclinical research. Circulation Research. 2015;116(1):116-26. DoI: https://doi.org/10.1161/CIRCRESAHA.114.303819

3. Baker M. 1,500 scientists lift the lid on reproducibility. Nature. 2016;533:452-4.

4. Silva LC. Crisis en la calidad de la ciencia médica: el papel del arbitraje en el nuevo desorden editorial. IRIS. Informação, Memória e Tecnologia. 2018;4(1):8-21.

5. Gøtzsche P. Deadly medicines and organized crime. How big pharma has corrupted healthcare. New York: Radcliffe, 2013.

6. Budapest Open Access Initiative. Ten years on from the Budapest Open Access Initiative: Setting the default to open. Budapest Open Access Initiative; 2012. Disponible en: http://www.budapestopenaccessinitiative.org/boai-10-recommendations

7. Nosek BA, Bar-Anan Y. Scientific communication is changing and scientists should lead the way. Psychological Inquiry. 2012;23(3):308-14.

8. Bosman, J. Nine reasons why impact factors fail and using them may harm science. I\&M / I\&O 2.0; 2013. Disponible en: https://im2punt0.wordpress.com/2013/11/03/nine-reasons-why-impact-factors-fail-and-using-them-may-harm-science/

9. Parra S. Auge, caída y auge del remdesivir, el "esperanzador" antiviral para combatir el covid-19; 2020. Disponible en: https://www.xataka. com/medicina-y-salud/auge-caida-auge-remdesivir-esperanzador-antiviral-para-combatir-covid-19

10. Gautret P, Lagier JC, Parola P, et al. Hydroxychloroquine and azithromycin as a treatment of CovID-19: Results of an open-label non-randomized clinical trial. Int J Antimicrob Agents; 2020:56(1): 105949. Dor: https://doi.org/10.1016/j.ijantimicag.2020.105949

11. Baker P, Rogers K, et al. Trump's aggressive advocacy of malaria drug for treating coronavirus divides medical community. The New York Times. 2020 abr. 6.

12. Mehra MR, Desai SS, Ruschitzka F, Patel AN. Retracted: Hydroxychloroquine or chloroquine with or without a macrolide for treatment of CovID-19: A multinational registry analysis. The Lancet, 2020(mayo 22):1-10. DoI: https://doi.org/10.1016/S0140-6736(20)31180-6

13. wHO Solidarity Trial Consortium. Repurposed antiviral drugs for COVID-19-interim WHO SOLIDARITY trial results. 2020; oct. 15. Disponible en: https://bit.ly/3nYAPhC 